\title{
Transverse single spin and azimuthal asymmetries in hadronic collisions at PHENIX
}

\author{
David Kleinjan ${ }^{1, a}$ \\ ${ }^{1}$ University of California, Riverside
}

\begin{abstract}
Inclusive transverse single spin asymmetries from high energetic polarized proton proton collisions provide insight into the spin-momentum correlations in QCD. Originally expected to be small in collinear perturbative QCD, results from PHENIX and other experiments show significant asymmetries in the forward momentum direction of the polarized proton over a wide range of center-of-mass energies. Several mechanisms have been proposed that attempt to explain these asymmetries, which include initial and final state effects. In order to disentangle these effects, a variety of probes is needed in different kinematic regions. In the PHENIX experiment at the Relativistic Heavy Ion Collider (RHIC), we study polarized $\mathrm{p}+\mathrm{p}$ collisions at center-of-mass energies up to $500 \mathrm{GeV}$. We will show transverse asymmetries at forward $(\$ 3.1<||$ eta $\mid<3.8 \$)$ and central rapidities $(\$||$ eta $\mid<0.35)$ and discuss their possible implications for intitial and final state effects.
\end{abstract}

\section{Introduction}

Transverse single spin asymmetries were initially predicted by pQCD to be small, on the order of $A_{N} \sim 10^{-4}$ [1]. However, the E704 experiment at Fermilab observed non-zero $A_{N}$ measurements for inclusive pion production at forward rapidity at $\sqrt{s}=19.2 \mathrm{GeV}$ [2]. These asymmetries have persisted at forward rapidity at various energies, including measurements made at RHIC by the BRAHMS, STAR, and PHENIX experiments [3-7], well into collision energy range of pQCD.

Since the non-zero $A_{N}$ results cannot originate from the hard-partonic scattering, spin-momentum correlations in initial and final state interactions, may explain these non-zero asymmetries. The Sivers Effect explains nonzero $A_{N}$ observables as a result of an intrinsic $k_{T}$ imbalance in the initial state parton correlated with the proton's spin $[8,9]$. This intrinsic $k_{T}$ imbalance leads to a bias in the transverse momentum of the hadron produced in the fragmentation of these quarks, which in turn leads to the non-zero transverse single spin asymmetry. The Collins Effect explains non-zero $A_{N}$ as originating from a correlation between a transversely polarized quark in the initial state to its spin dependent fragmentation function in the final state, i.e. the Collins Fragmentation Function [10]. Due to the initial transverse spin of the quark in the initial state, the $p_{T}$ of the leading hadron in the final state Collins fragmentation has a non-zero azimuthal dependency with respect to this initial transverse quark spin of the quark.

In addition to the spin-momentum correlations, collinear higher-twist effects (twist-3) explain non-zero $A_{N}$ results by the interference between a gluon field in the initial or final states within the collinear factorization

\footnotetext{
a e-mail: david.kleinjan@email.ucr.edu
}

limit. While these correlation functions do not contain direct information on the transverse momentum distributions of partons, this approach has been related to $k_{T}$-moments of TMD PDFs and FFs such as the Sivers and Collins functions for multiparton correlations in the initial and final state, respectively [11]. Prior RHIC transverse SSA measurements for inclusive hadron production have been described relatively well by a combination of twist- 3 effects in the initial and final states [12-15].

This proceedings reports on transverse spin measurements at central and forward rapidity for $\pi^{0}$ and $\eta$ mesons, and forward rapidity $J / \psi$ and single muons as measured by the PHENIX detector at RHIC.

\section{The PHENIX Detector}

The PHENIX detector [16] has a high rate capability utilizing a fast DAQ and specialized triggers, high granularity detectors, and good mass resolution and particle identification. The PHENIX detector consists of two spectrometer arms at central rapidity $|\eta|<0.35$, two Muon arms at near forward rapidity $1.2<|\eta|<2.4$, two global detectors, and two calorimeters at forward rapidity $3.1<|\eta|<3.9$.

The central arm spectrometers contain finely grained electromagnetic calorimeters, which are used to measure $\pi^{0}$ and $\eta$ mesons $A_{N} . J / \psi$ mesons are measured via decay into muons and detected using the Muon Arms.

The forward calorimeters are called the MPC detectors in PHENIX. Each MPC is made of around $2002.2 \times 2.2 \times$ $18 \mathrm{~cm}^{3} \mathrm{PbWO}_{4}$ crystal towers. The MPC is capable of reconstruction di-photon $\pi^{0}(\eta)$ mesons in an energy range of 7-20 (10-70) GeV. For $\pi^{0}$ mesons with an energy greater than $20 \mathrm{GeV}$, the two photons are merged beyond the resolution of the MPC, but they can still be identified as single 


\section{$p+p \quad \sqrt{s}=200 \mathrm{GeV}$}

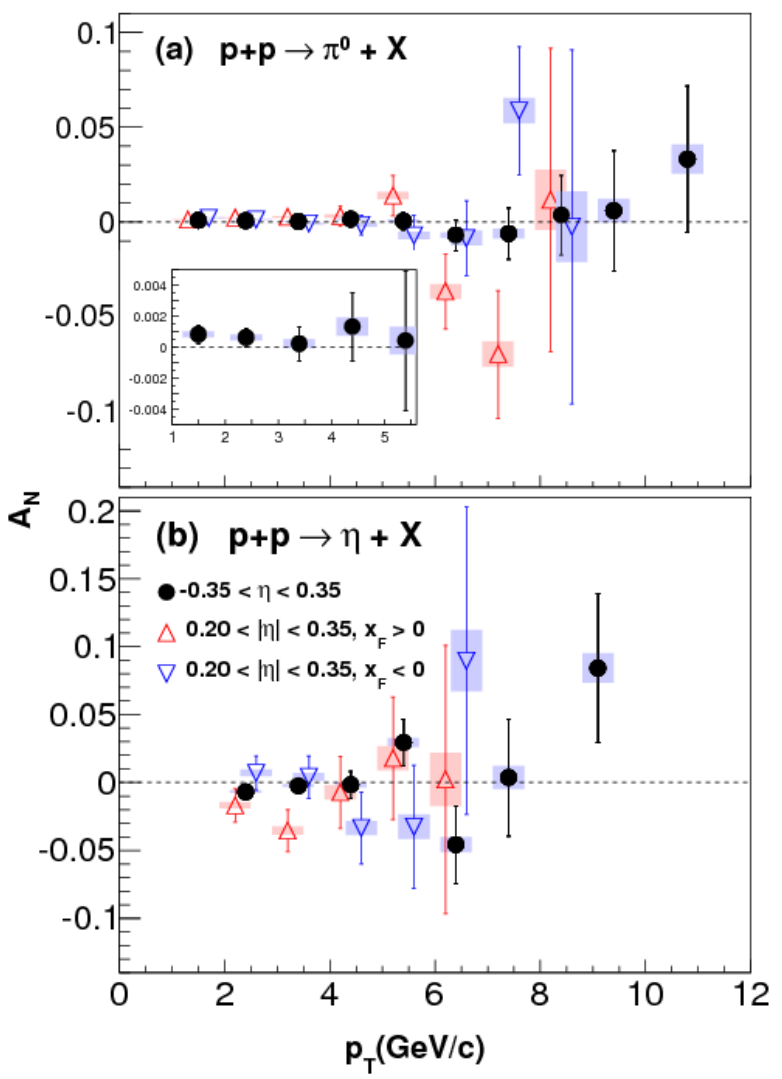

Figure 1. The central rapidity $\pi^{0}$ and $\eta$ meson $A_{N}$ at $\sqrt{s}=200 \mathrm{GeV}[6]$

clusters. Using a Monte-Carlo simulation, $80 \%$ of clusters with $p_{T} \leq 3 \mathrm{GeV} / c$ are identified as merged $\pi^{0} \mathrm{~s}$, decreasing to $70 \%$ at higher $p_{T}$ as high energy direct photons begin to contribute more to the cluster composition.

\section{Mid-Rapidity measurements of $\pi^{0}$ and $\eta$ meson $A_{N}$}

Figure 1 shows the $A_{N}$ for $\pi^{0}$ and $\eta$ mesons at central rapidity as measured by the PHENIX central arms [6], which has a factor of 20 improvement on the previous $\pi^{0}$ results [17]. The $\pi^{0}$ and $\eta$ meson asymmetry measurements are consistent with zero. The dominant fragmentation process at central rapidity is from gluons, putting constraints on the gluon Sivers function [18].

\section{Forward measurements of $\pi^{0}$ and $\eta$ meson $A_{N}$ using the MPC detector}

\section{1 $A_{N}$ of $\pi^{0}$ mesons and single clusters}

Results of the $A_{N}$ of $\pi^{0}$ at $\sqrt{s}=62.4 \mathrm{GeV}$ measured in PHENIX using the MPC detectors is shown in Fig. 2 [6]. The top panel shows a comparison to other $\pi^{0} A_{N}$ at various energies, where it is demonstrated that the asymmetry shows little dependence on $\sqrt{s}$. The bottom panel shows
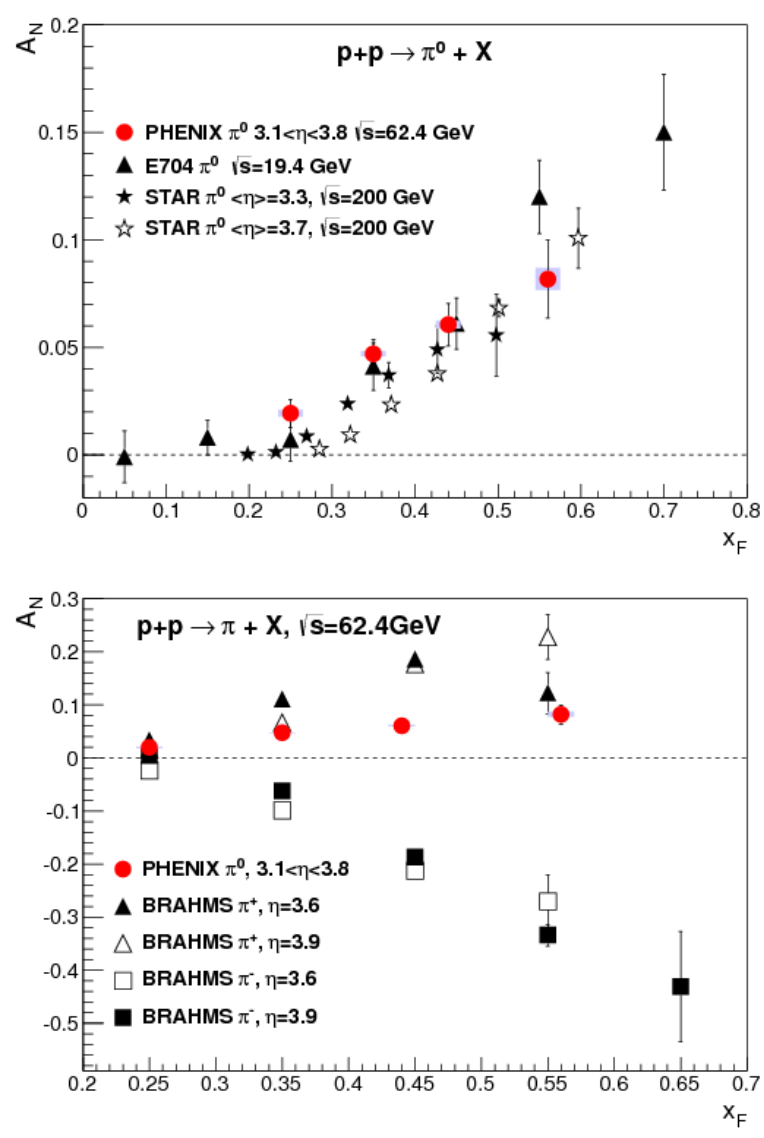

Figure 2. $A_{N}$ at forward rapidity for $\pi^{0}$ mesons at $\sqrt{s}=$ $62.4 \mathrm{GeV}$ [6]. (top) Comparison to other forward rapidity $\pi^{0}$ $A_{N}$ measurments. (bottom) Comparison to charged pions at the same energy.

a comparison to charge pions at the same energy as measured by BRAHMS [3]. These asymmetries were compared to studies of the $\pi^{ \pm, 0}$ fragmentation functions done using Pythia. The $\pi^{+-, 0}$ were studied in Pythia to determine which valence quark they originated from, and it was found that the $\pi^{+}$comes primarily from the $u$ quark, the $\pi^{-}$ comes half the time from a $u$ quark, and half the time from a $d$ quark. The $\pi^{0}$ comes $3 / 4$ of the time from a $u$ quark and $1 / 4$ of the time from a $d$ quark. Assuming that one can accurately determine the quark origins for the pions from Pythia, this suggests that the pion $A_{N}$ s cannot be dominantly from the Sivers effect.

Because of $\pi^{0}$ merging effects, $A_{N}$ for single clusters at $\sqrt{s}=200 \mathrm{GeV}$ is measured, and $\pi^{0}$ interpretations are made based on the simulations of single clusters, mentioned in Section 2. Comparison of PHENIX cluster $A_{N}$ is made with the STAR $\pi^{0} A_{N}$ at the same energy and using the same dataset in Fig. 3. Here $A_{N}$ is plotted vs $p_{T}$, binned in $x_{F}$. At lower $x_{F}$, where simulations indicate that PHENIX clustering is dominated by merged $\pi^{0} \mathrm{~s}$, there is good agreement with STAR. At higher $x_{F}$ there is some tension between the results, $A_{N}^{\text {PHENIX }} \lesssim A_{N}^{S T A R}$. Based on the Monte-Carlo simulations, direct photons may have a non negligible effect on the PHENIX cluster $A_{N}$ at high- 


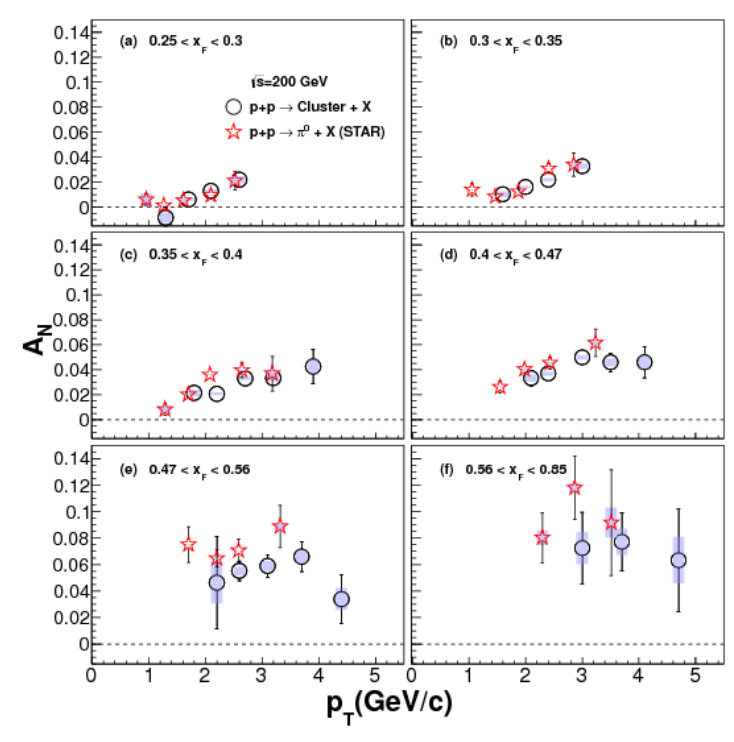

Figure 3. Comparison of $A_{N}$ of electromagnetic clusters and $\pi^{0}$ mesons at $\sqrt{s}=200 \mathrm{GeV}[4,6]$.

$p_{T}$, suggesting $A_{N}^{\gamma} \lesssim A_{N}^{\pi^{0}}$. Any future measurement of the direct photon $A_{N}$ would be interesting to confirm or deny this assumption.

Measurements of $\pi^{0}$ and cluster $A_{N}$ as a function of transverse momentum $\left(p_{T}\right)$ are are done because certain interpretations of the twist- 3 effects predict that $A_{N}$ decreases with increasing $p_{T}$ on the same order as the partonic momentum scale $Q^{2}$. Where this turnover occurs is unknown. Figure 3 demonstrates that no sign of decreasing $A_{N}$ has been observed for inclusive $\pi^{0} \mathrm{~s}$ (cluster) at forward rapidity within statistical uncertainty.

\section{$4.2 A_{N}$ of $\eta$ mesons}

Results of the $A_{N}$ for $\eta$ mesons at $\sqrt{s}=200 \mathrm{GeV}$ are shown in Fig. 4. The top panel shows $A_{N}$ as a function of $x_{F}$. There is a 2 to $20 \%$ asymmetry across forward $x_{F}$, and the average value of backwards $x_{F}$ points are consistent with zero with $1.7 \sigma$. The bottom panel shows $A_{N}$ as a function of $p_{T}$ for forward and backwards $x_{F}$. The $p_{T}$ dependence of $A_{N}$ at forward $x_{F}$ is compared with a twist-3 calculation [13], which describes the magnitude of the asymmetry well at the lowest and highest points in $p_{T}$, but it is unclear whether the observed shape for the middle $p_{T}$ values is well described. However, the theoretical uncertainty is not available for the twist- 3 calculation, and this new $\eta$ meson $A_{N}$ result will help disentangle various twist-3 interpretations.

Figure 5 shows a comparison to other $\pi^{0}$ and $\eta$ meson results. The Fig shows a comparison with previous $\pi^{0}$ meson measurements, and suggests that the $\eta$ meson asymmetry is consistent with previous $\pi^{0}$ meson asymmetries, where differences could have arisen due to isospin and mass differences, and the fragmentation process (e.g. strangeness contribution for the $\eta$ meson). The bottom

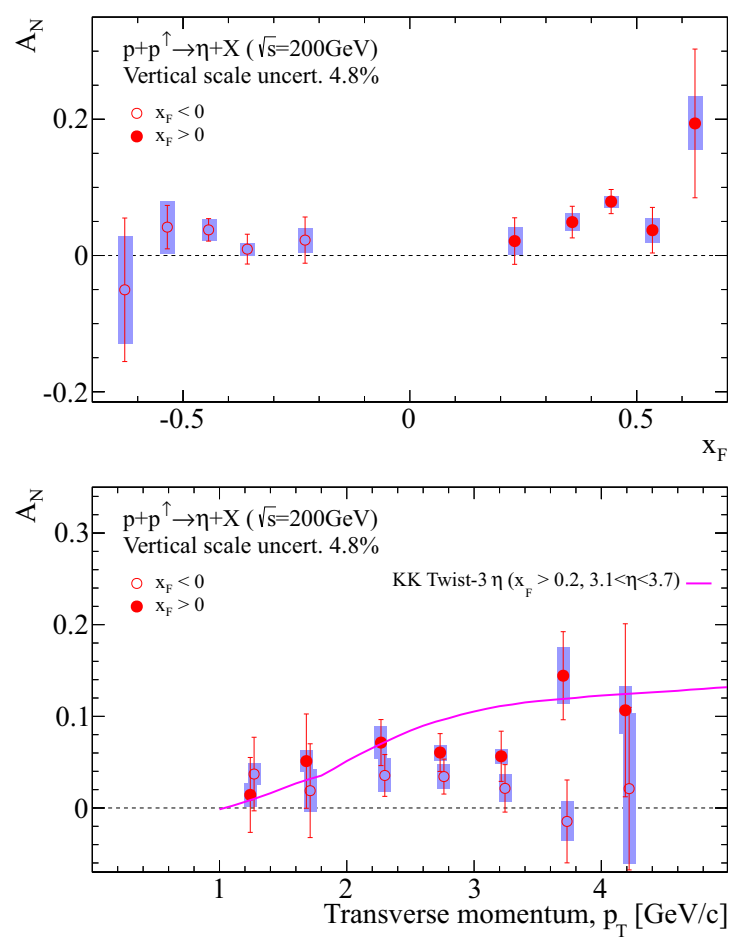

Figure 4. $A_{N}$ at forward rapidity for $\eta$ mesons at $\sqrt{s}=62.4 \mathrm{GeV}$ [7]. (top) $x_{F}$ dependence of $A_{N}$. (bottom) $x_{F}$ dependence of $A_{N}$.

panel shows a comparison to previous $\eta$ meson $A_{N}$, including the measurement made by STAR at the same collision energy. The results are consistent between PHENIX and STAR within uncertainties.

\section{Forward measurements of $A_{N}$ for heavy flavor}

The forward muon detectors have been used to measure the $A_{N}$ of $J / \Psi$ mesons, and is shown in the top panel of Fig. 6. Only the color singlet generates the $A_{N}$, so the measurement is sensitive to this production mechanism. The $A_{N}$ of $J / \Psi$ mesons is consistent with zero, but is currently limited by statistics. The bottom panel shows single muon $A_{N}$, which is sensitive to heavy flavor $D$ meson decay. $D$-meson is dominated by gluon-gluon fusion, therefore heavy flavor $A_{N}$ is sensitive solely to the gluon Sivers function. This $A_{N}$ is consistent with zero.

\section{Future}

Both $J / \Psi$ and heavy flavor $A_{N}$ will be improved by a forward silicon vertex detector (FVTX), which was commissioned during a large 2012 transverse spin data at $\sqrt{s}=200 \mathrm{GeV}$. In addition, 2015 data sample at RHIC with a fully commissioned FVTX will greaty enhance these measurements statistically by rejecting hadron decay background.

Also, a preshower upgrade to the forward MPC electromagnetic calorimeter approved, and will begin taking data in 2015. The MPC Extension Detector (MPC-EX) 

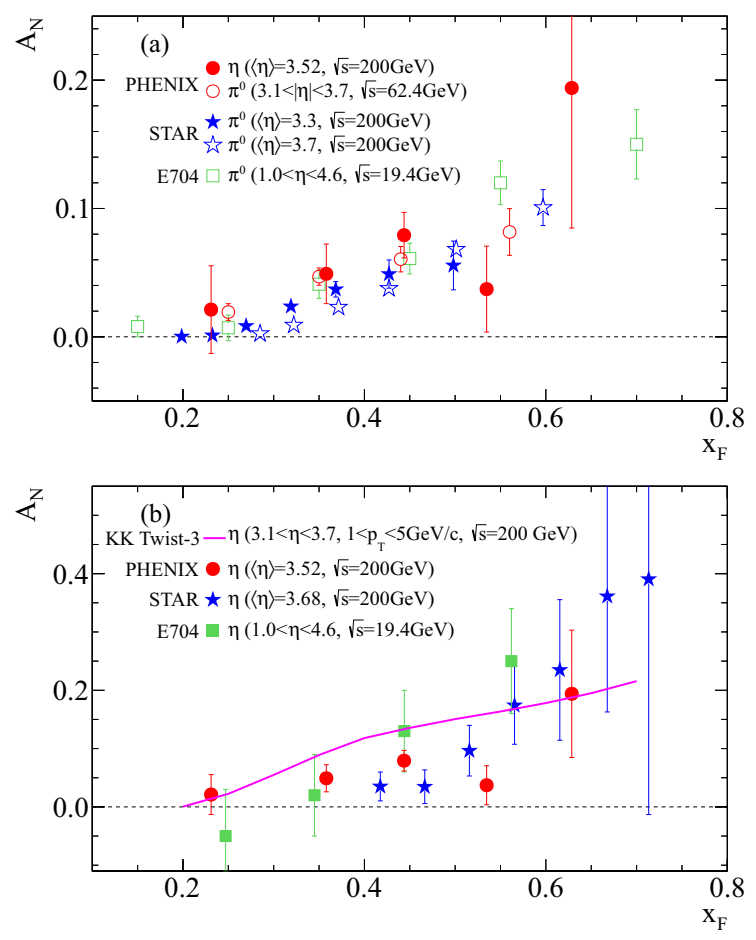

Figure 5. Comparison of PHENIX $A_{N}$ of $\eta$ mesons to other $A_{N}$ measurements. (top) Comparison to other $\pi^{0} A_{N}$ measurements. (bottom) Comparison to other $\eta$ meson $A_{N}$ measurements.
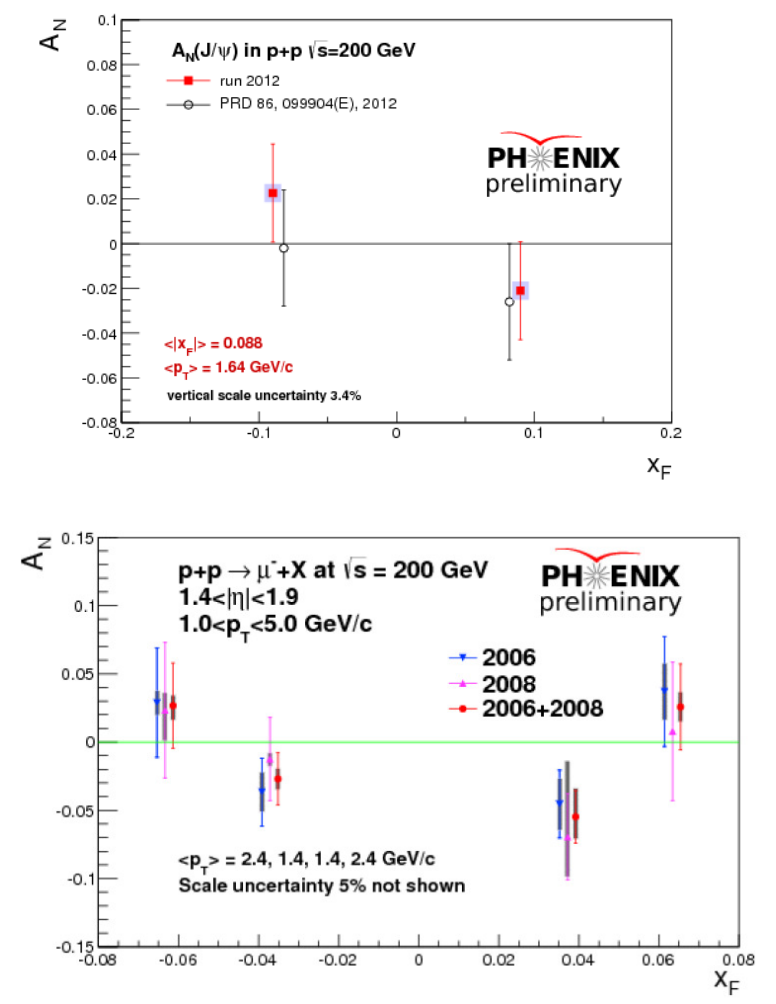

Figure 6. $x_{F}$ dependence of $A_{N}$ for the $J / \psi$ (top) and single muon (bottom). is an eight layer silicon minipad tungsten sandwich preshower detector installed in front of the existing electromagnetic calorimeter MPC. The MPC-EX in coincidence with the MPC-EM will allow for the measurement of isolated direct photons, thus measurement of direct photon $A_{N}$. Free from the Collins Effect since the direct photon process does not involve a fragmentation function, direct photon $A_{N}$ is isolated to studying the Sivers Effect and twist-3 quark gluon distribution functions.

\section{Conclusion}

The PHENIX detector has measured transverse single spin asymmetries for $\pi^{0}$ and $\eta$ mesons at central and forward rapidity, and for $J / \psi$ and single muons at forward rapidity. Central rapidity measurements of $\pi^{0}$ and $\eta$ mesons constrain the gluon Sivers effect, while forward $\pi^{0}, \eta, J / \psi$ and single muon results provide information to disentangle Collins, Sivers, and twist-3 effects. Upgrades at PHENIX include the FVTX detector to improve $J / \psi$ and heavy flavor $A_{N}$, and the MPC-EX detector will provide a first measurement of direct photon $A_{N}$ at forward rapidity.

\section{References}

[1] G.L. Kane, J. Pumplin, W. Repko, Phys. Rev. Lett. 41, 1689 (1978)

[2] D. Adams et al., Phys. Lett. B264, 464 (1991)

[3] I. Arsene et al. (BRAHMS Collaboration), Phys. Rev. Lett. 101, 042001 (2008)

[4] B.I. Abelev et al. (STAR Collaboration), Phys. Rev. Lett. 101, 222001 (2008)

[5] L. Adamczyk et al. (STAR Collaboration), Phys. Rev. D 86, 051101 (2012)

[6] A. Adare et al. ((PHENIX Collaboration)), Phys. Rev. D 90, 012006 (2014)

[7] D. Kleinjan et al., http://arxiv.org/abs/14063541

[8] D. Sivers, Phys. Rev. D 41, 83 (1990)

[9] D. Sivers, Phys. Rev. D 43, 261 (1991)

[10] J. Collins, Nucl. Phys. B396, 161 (1993)

[11] X. Ji, J.W. Qiu, W. Vogelsang, F. Yuan, Phys. Rev. Lett. 97, 082002 (2006)

[12] K. Kanazawa, Y. Koike, Phys. Rev. D 82, 034009 (2010)

[13] K. Kanazawa, Y. Koike, Phys. Rev. D 83, 114024 (2011)

[14] A. Metz, D. Pitonyak, Phys. Lett. B 723, 365 (2013)

[15] K. Kanazawa, Y. Koike, A. Metz, D. Pitonyak, arXiv:1404.1033

[16] K. Adcox et al., Nucl. Instrum. Meth. A499, 469-479 (2003)

[17] S. Adler et al. (PHENIX Collaboration), Phys. Rev. Lett. 95, 202001 (2005)

[18] M. Anselmino, U. D’Alesio, S. Melis, F. Murgia, Phys. Rev. D 74, 094011 (2006) 